Journal of Education and Science (ISSN 1812-125X), Vol: 30, No: 2, 2021 (67-78)

\title{
Investigation of Important Fatty Acids in Biofuel Production from Number of Microalgae
}

\author{
Taha A. Kh. Al-Someidae ${ }^{1 *}$, Yousif J. Al-Shahery ${ }^{2}$, Qutaiba Shuaib Al-Nema ${ }^{3}$ \\ ${ }^{1 * 2,3}$ Department of Biology, College of Education of Pure Science, University of Mosul, Mosul, Iraq \\ E-mail: ²yousefalshahery@uomosul.edu.iq, ${ }^{3}$ dr.qutaibashuaib@uomosul.edu.iq
}

(Received October 10, 2020; Accepted December 14, 2020; Available online June 01, 2021)

DOI: 10.33899/edusj.2020.128619.1115 @ 2021, College of Education for Pure Science, University of Mosul.

This is an open access article under the CC BY 4.0 license (http://creativecommons.org/licenses/by/4.0/).

\begin{abstract}
Algae biofuels is considered as an alternative source to fossil fuels. In recent decades, there was a significant increase in the use of energy sources in order to avoid the depletion of traditional sources such as coal and petroleum. The produced fuel from algal oil had important characteristics compared to that from other vegetable crops. This is due to the short life cycle of development, a fast-growing and easy to be developed. In this study, three types of micro-algae Scendesmus dimorphus, Chlorella vulgaris and Chlorococcum humicola were used and grown in in a 5 liter photobioreactor. The dry biomass productivity of the three algae was estimated, and then a chemical analysis of the total fatty was performed to detect their biological contents as well as diagnose the fatty acid. Results showed that S. dimorphus produced the highest levels in both biomass, $1.58 \mathrm{~g} \mathrm{l}^{-1}$ from dry weight and estimation of the total fat indicated $C$. vulgaris has the highest total fat yield, at $29.6 \%$. Results of fats characterization using (GLC) showed that S. dimorphus produced the high percentage of saturated fatty acids for the meristic acid ester (C14: 0) by 47\% and the lincoseric acid ester (C24: 0) was 7.194\%. In contrast, both Chlorella vulgaris and Chlo. humicola showed less level of saturated fatty acids. This indicates the suitability of algae oil derived from $S$. dimorphus in the synthesis of fatty acid, a major source in producing biofuels.
\end{abstract}

Keywords: S. dimorphus, Chlo. humicola, C. vulgaris, Microalgae, Fatty acids, Biofuel.

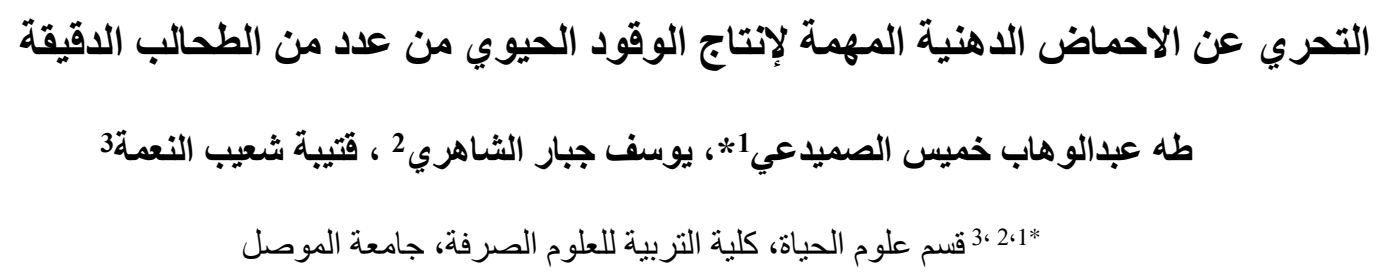


الخلاصة

يُعد وقود الطحالب الحيوي من المصادر الواعدة كبديل عن الوقود الاحفوري، وتحسباً لنفاذ مصادر الطاقة التقليدية كالفحم والبترول فقد ازدادت في العقود الاخيرة الحاجة الملحة للبحث عن مصادر جديدة للطاقة. اتسم الوقود المنتج من الزيت الطحلبي

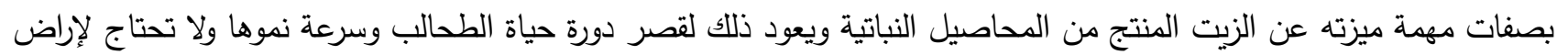

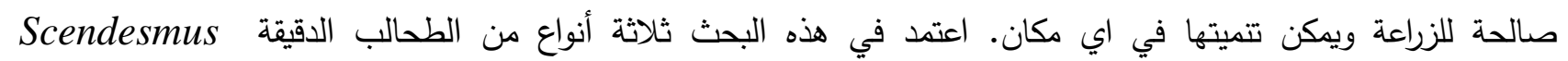
Chlorococcum humicola و Chlorella vulgaris،dimorphus وقدرت انتاجيتها من الكتلة الحيوية الجافة ثم اجري التحليل الكيميائي لتحديد محتواها الحيوي من الدهون الكلية وتشخيص الاحماض الدهنية. وبينت النتائج ان اعلى إنتاجية للكتلة الحيوية كانت في الطحلب S. dimorphus بلغت 1.58 غم لتر -1 من الكتلة الحيوية الجافة. في حين أشارت نتائج تقدير الدهون حصول الطحلب C. vulgaris على أعلى إنتاجية من الدهون الكلية وقد بلغت 29.6 \%. اما فيما يخص نتائج التشخيص باستخدام تقنية GLC فقد وجدت أن أعلى نسبة من الاحماض الدهنية المشبعة من زيت الطحلب S. dimorphus كانت لأستر الحامض المرستيك (C14:0) بنسبة 47.105 \% وأستر الحامض اللينكوسيريك (C24:0) بنسبة 7.194 \% في حين اظهر الطحلب Chlo. humicola velgaris والطحلب مستوى اقل من الاحماض

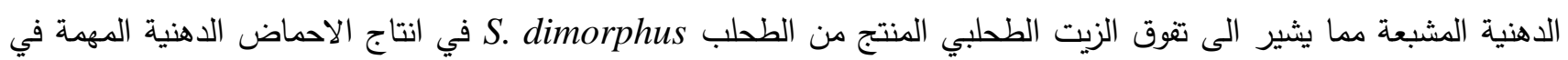
تشكيل الوقود الحيوي.

الكلمات المفتاحية: Chlo.humicola ، C. vulgaris ،S. dimorphus، الطحالب الدقيقة، الاحماض دهنية، الوقود الحيوي.

\section{Introduction المقدمة}

ازدادت في العقود الاخيرة الحاجة الملحة للبحث عن مصادر جديدة للطاقة تحسبا لنفاذ مصادر الطاقة التقليدية كالفحم والبترول, فضلا عن تأثيرها الواضح والخطير للانبعاثات الكيميائية الغازية الضارة على حياة الكائنات الحية. لهذا تركزت الابحاث العالمية للبحث عن مصادر للوقود صديقة للبيئة واقتصادية التكلفة وقابلة للتجدد. حيث اكتثفت العديد من مصادر الطاقة المتجددة

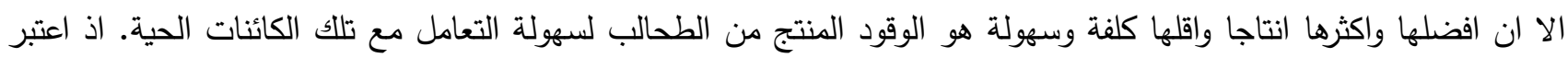

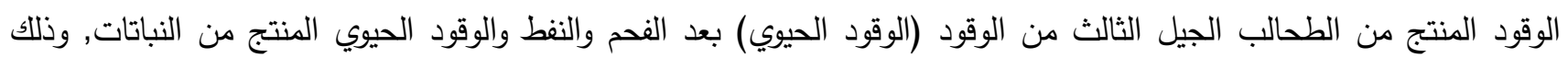
لتميزها عن باقي المصادر الحيوية الاخرى اذ ان الوقود الحيوي المنتج من الذرة ,قصب السكر , بذور اللفت ، فول فود الصوديا والنخيل لا يمكن استخدامها في المحركات المعدلة ولا تتطبق على مواصفات التسويق فضلا عن ذلك تعتبر من المحاصيل الغذائية المهمة

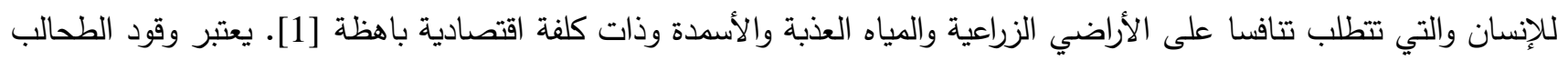
الحيوي (Algal Biodiesel) من المصادر الواعدة كبديل عن الوقود الاحفوري والحيوي الاخرى والتي يمكن أن تتمو في المياه

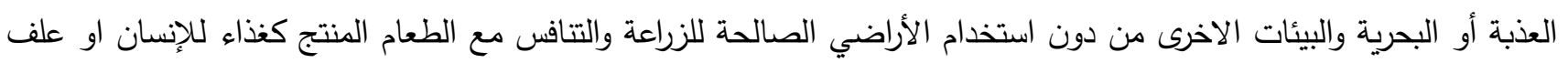

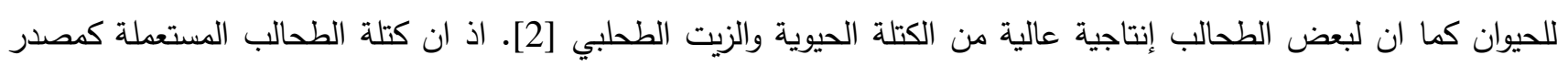
للجيل الثالث من الوقود الحيوي ذات إنتاجية أعلى من مصادر الجيل الثاني التي تصل إلى من حوالي 30 ضعفاً من الانتاجية لكل

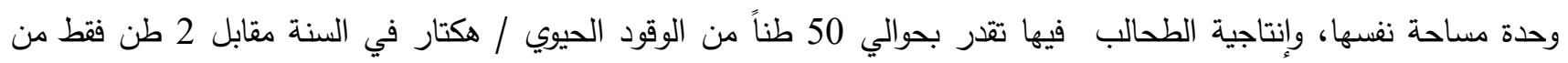

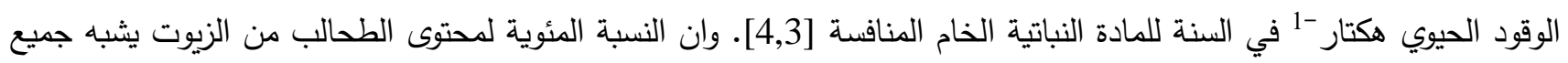

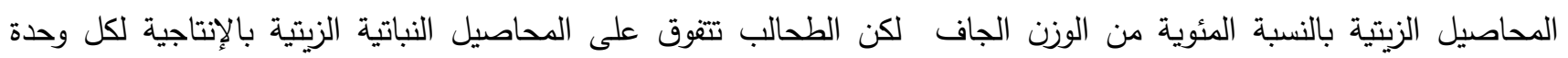
مساحة نفسها وبالإمكان مضاعفة الإنتاجية نتيجة لقصر دورة حياة الطحالب والتي تتضاعف خلانس خلال 24 ساعة مقارنة بطول دورة 
حياة النباتات[5]. علاوة على ذلك ، فإن عملية إنتاج الوقود الحيوي من الجيل الأول مسؤولة أيضًا عن تدهور البيئة، لذلك تلاشت التي

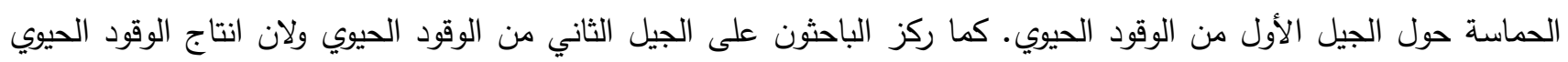

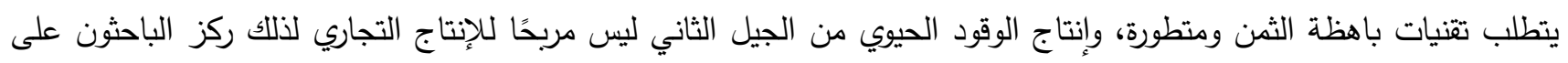

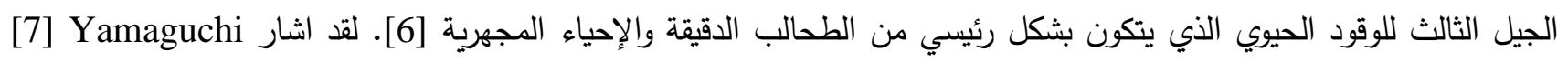

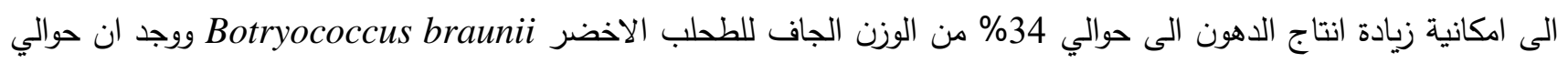

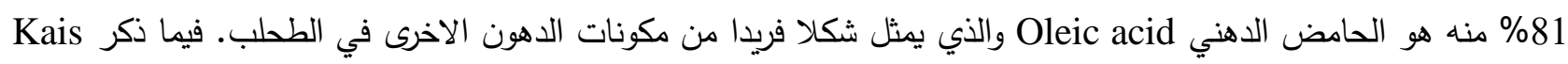
[8] امكانية استخدام الطحالب الدقيقة مثل Botryococcus و Chlorella لإنتاج الوقود الحيوي السائل لحل ازمة الطاقة،

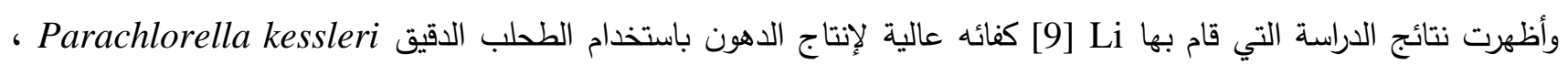
واوضح Ahmed [10] في دراسته امكانية تحويل واسترة الدهون المستخلصة من الطحالب الدقيقة Chlorella و Rhizoclonium Botryococcus و Mohamed ، كما ركزت دراسة Almutairi [12] على العديد من الظروف والمتغيرات لإنتاج الوقود الحيوي من الطحلب المجهري

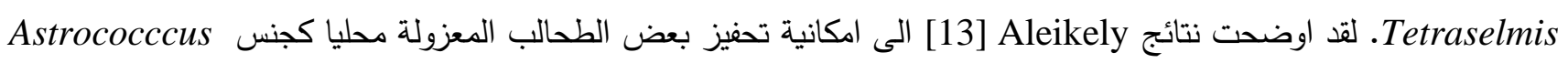
ق Chlorococcum humicola و لإنتاج الوقود الحيوي. وتهدف الدراسة الحالية للكثف عن و sp. قابلية بعض الطحالب الدقيقة الثائعة في المياه العذبة لإنتاج الاحماض الدهنية المهمة في تثكيل الوقود الحيوي.

Materials and Methods مواد وطرائق العمل الكائن المجهري وظروف الزراعة : تم الحصول على الطحالب الخضراء الدقيقة قيد الدراسة من عدة مصادر كما في الجدول (1) : الجدول (1): الاجناس الطحلبية قيد الدراسة.

\begin{tabular}{|c|c|c|}
\hline 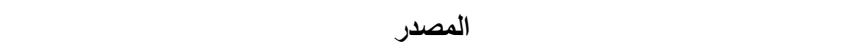 & 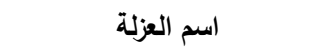 & ت \\
\hline مختبر الطحالب كلية العلوم/ جامعه المنصورة/ جمهورية مصر العربية & Scendesmus dimorphus & 1 \\
\hline وحدة بايوتكنلوجيا الطحالب في المركز القومي للبحوث//القاهرة/جمهورية مصر العربية & Chlorella vulgaris & 2 \\
\hline كلية التربية للعلوم الصرفة ابن الهيثم / جامعة بغداد / العراق & Chlorococcum humicola & 3 \\
\hline
\end{tabular}

وسط تنمية الطحلب الدقيقة:-

حضر وسط Chu-13 كما مبين في الجدول (2) وضبط الاس الهيدروجيني عند 7.5، ولتحضير اللقاح وزع الوسط الزرعي على دوارق زجاجية مختلفة الاحجام بنسبة حجمية 20\% ثم عقدت بجهاز المؤصدة عند درجه حرارة 121 درجة

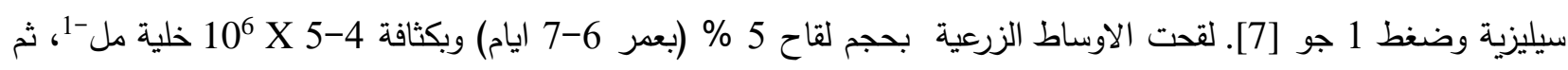
حضنت في حاضنة هزازة عند درجه حرارة 25 سيليزية ومعدل رج 100 رجة دقيقة-1، بينما تم تجهيز المفاعل الضوئي سعة 5 لتر لغرض انتاج الكتلة الحيوية بمصدر هواء خارجي معقم لغرض التهوية والتقليب باستخدام فلتر ملي بور ذو فتحات 0.45 مايكرومتر وباستخدام مضخة هواء صغيرة (شكل 1) وتم تعقيم الوسط بحجم 3 لتر وتلقيح وتحضين المفاعل الضوئي بنفس الشروط المذكورة اعلاه . 
الجدول (1): تراكيز المواد الكيميائية للوسط 13 Chu.

\begin{tabular}{|c|c|c|c|}
\hline الحجم المضاف بالمل لتحضير واحد & التركيز النهائي & 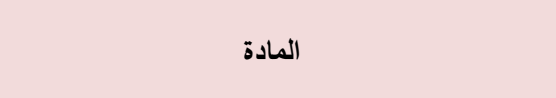 & $ت$ \\
\hline 10 & 400 & $\mathrm{KNO}_{3}$ & 1 \\
\hline 10 & 80 & $\mathrm{~K}_{2} \mathrm{HPO}_{4}$ & 2 \\
\hline 10 & 107 & $\mathrm{CaCL}_{2}$ & 3 \\
\hline 10 & 200 & $\mathrm{MgSO}_{4} .7 \mathrm{H}_{2} \mathrm{O}$ & 4 \\
\hline 10 & 100 & Citric acid & 5 \\
\hline 10 & 20 & Ferric citrate & 6 \\
\hline & & $\begin{array}{l}\text { Micro elements } \\
\text { العناصر الصغرى }\end{array}$ & \multirow{7}{*}{7} \\
\hline 1 & 5.720 & $\mathrm{H}_{3} \mathrm{BO}_{3}$ & \\
\hline 1 & 0.020 & $\mathrm{CoCl}_{2}$ & \\
\hline 1 & 0.440 & $\mathrm{ZnSO}_{4} .7 \mathrm{H}_{2} \mathrm{O}$ & \\
\hline 1 & 0.160 & $\mathrm{CuSO}_{4} .5 \mathrm{H}_{2} \mathrm{O}$ & \\
\hline 1 & 0.084 & $\mathrm{NaMoO}_{4}$ & \\
\hline 1 & 3.620 & $\mathrm{MnCl}_{2}$ & \\
\hline 1 1 قطرة & & قطرة من حامض الكبريتيك بتركيز 0.072 عياري لتر -1 & 8 \\
\hline
\end{tabular}

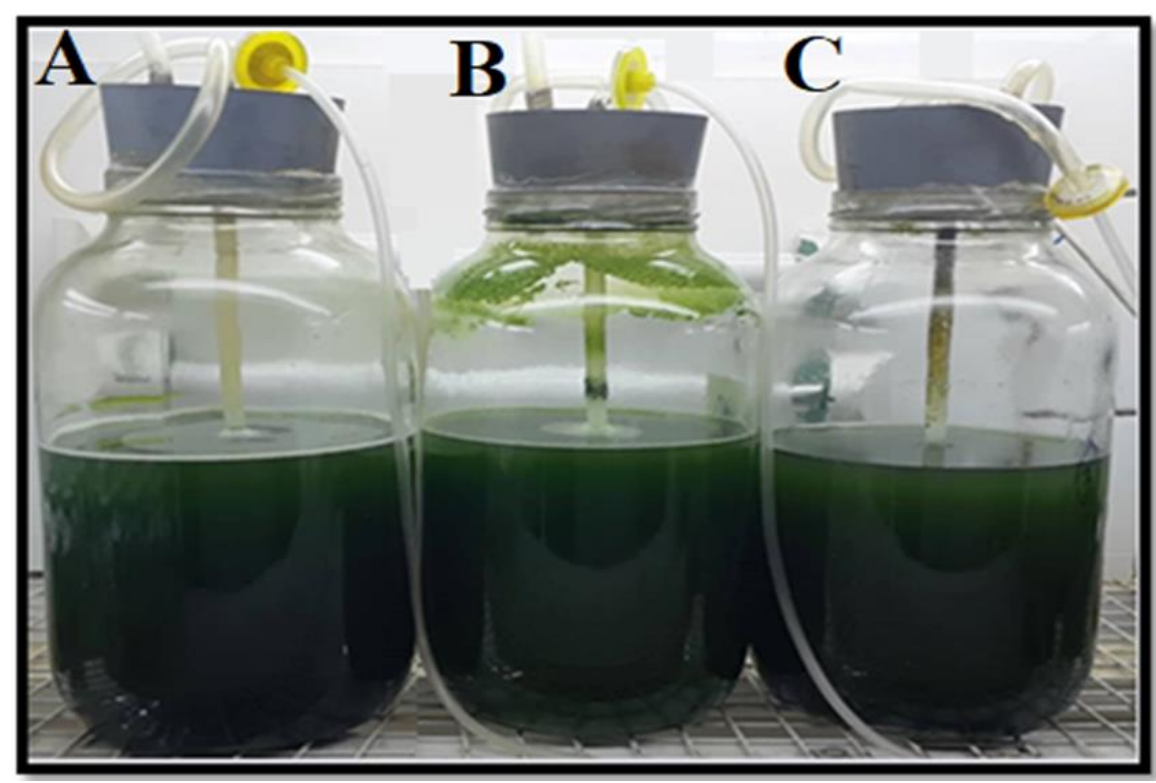

الثكل (1): المفاعل الضوئي سعة 5 لتر بعد التلقيح بالطحالب والنمو بعر 25 يوم، A: مزرعة الطحلب Scendesmus،

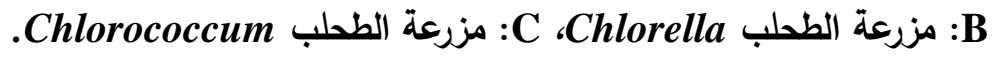
حصاد خلايا الطحالب :استخدمت في هذا البحث طريقتي الترسيب بالجاذبية مدعومة بطريقة الطرد المركزي عند 9000 دورة دقيقة-1 لمدة 5 دقائق لترسيب ما تبقى من الخلايا الطحلبية في الوسط الزرعي [14]. تقدير الدهون الكلية : اعتمد في هذا البحث مزيج الهكسان / الأيزوبروبانول (2/3 ح / ح ) والوقت المستخدم للاستخلاص هو 7.5 ساعة (450

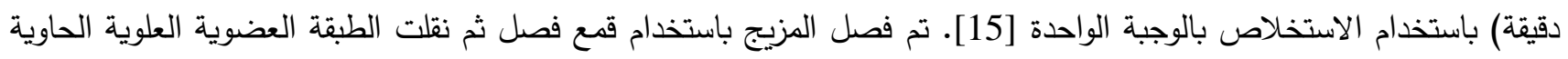
للدهون إلى قنينة زجاجية نظيفة وجافة موزونة مسبقا (W1)، وتم تبخير العينة في فرن الهواء الساخن عند 80 درجة مئوية لمدة

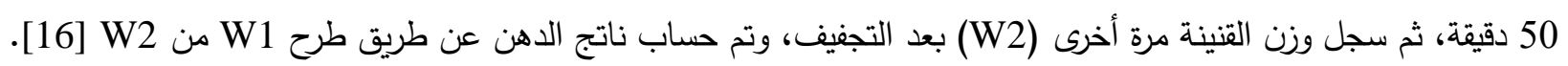
الحصول على الأحماض الدهنية بثكلها الحر :- 
تم الحصول على الاحماض الدهنية بشكل حر من خلال أجراء عملية الصوبنة للدهون الكلية في وسط قاعدي وفقاً لطريقة . [17] Arthur

تحضير المثيل استر (الاسترة) Esterification أضيفت مجموعة المثيل إلى الحامض الدهني المفصول بعملية الصوبنة للتحويل إلى حالة اقل قطبية وجعله أكثر قابلية للتطاير

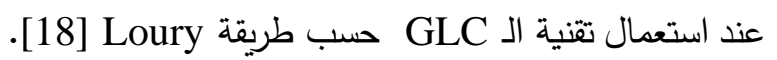

تشخيص الاحماض الاهنية باستخدام جهاز كروماتوكرافيا الغاز - السائل (GLC) :-

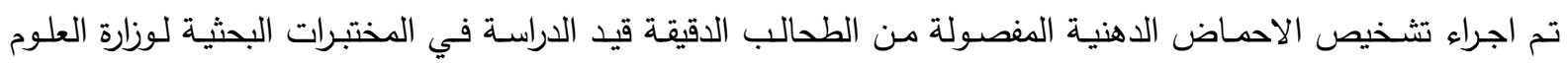

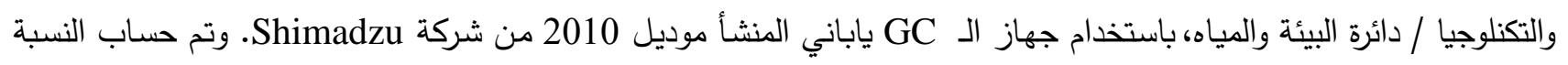
المئوية لكل حامض دهني في العينة من خلال المعادلة الأتية:-

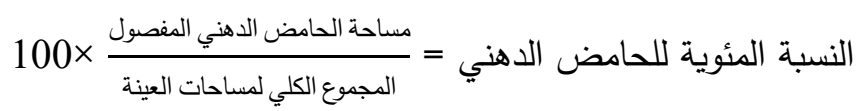

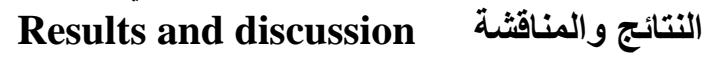
الحصاد وأنتاج وتقدير الكتلة الحيوية لأنواع الطحالب الدقيقة:-

تم حصاد الخلايا بطريقتي الترسيب بالجاذبية والطرد المركزي وجمعت الكتلة الحيوية الرطبة بعد الحصاد وجفقت ثم طحنت لادئ لغرض زيادة المساحة السطحية للمعاملات اللاحقة. وقيست الكتلة الحيوية لكل طحلب (الجدول 3). بينت العديد من الدراسات أهمية حصاد الطحالب بإزالة كميات كبيرة من الماء من المزرعة لزيادة التركيز للكتلة الحيوية من خلاد الترسيب اعتمادًا على حجم الطحالب الدقيقة [19]، وكان الغرض من ضبط ظروف النمو إنتاج أكبر كمية من الكتلة الحيوية التي ممكن أن تتراكم عندها الدهون، وبينت النتائج أن فترة التحضين (25) المعتمدة كانت مناسبة لتصل الخلايا في نموها الى طور الثبات والتي أعطت كتلة

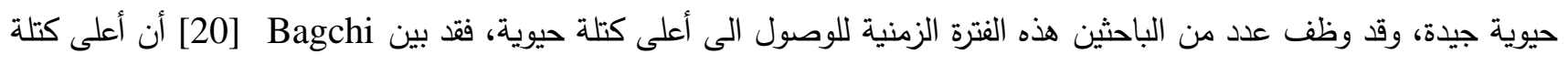

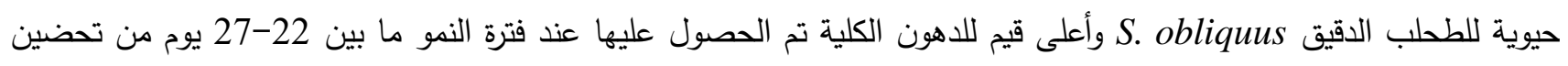

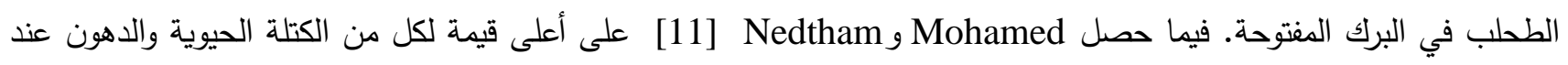
دراسة تأثير عدة عوامل على نمو الطحلب الدقيق Botryococcus braunii عند 25 يوماً من التحضين. وبينت النتائج أن أكبر قيمة للكتلة الحيوية الجافة كانت للطحلب الدقيق S. dimorphus وبلغت 1.58 غم لتر -1، في حين أن الكتلة الحيوية الجافة

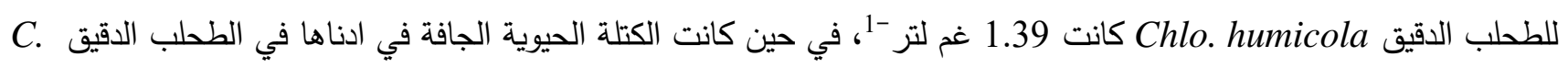
0.91 vulgaris

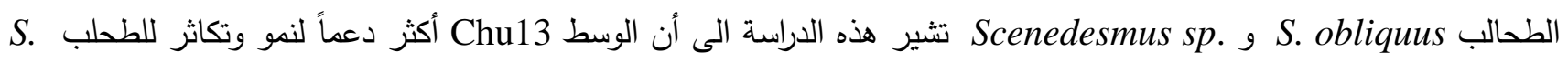

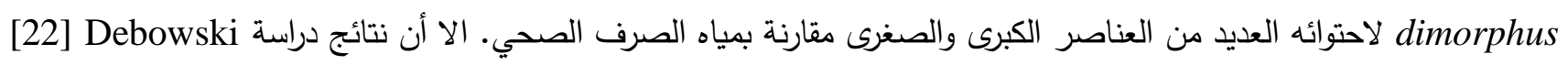

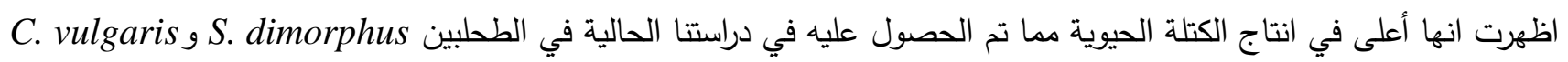

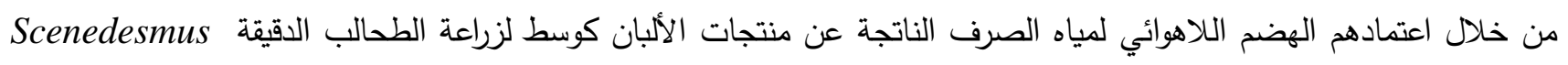
Chlorella sp. sp. الدراسة الحالية نتائج مقاربة نوعا ما لما حصل عليه Ferro م [21] من الكتلة الحيوية للطحلب C. vulgaris والتي بلغت 1.15 غم لتر -1. اهتمت الكثير من الدراسات بالطحالب الخضراء الدقيقة ذات القدرة العالية لإنتاج الكتلة الحيوية، وهي أكثر الكائنات

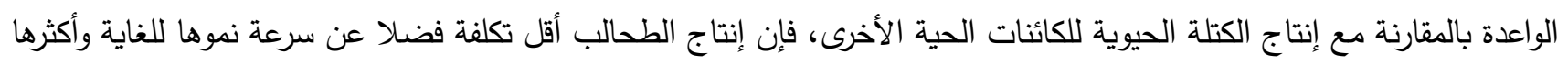

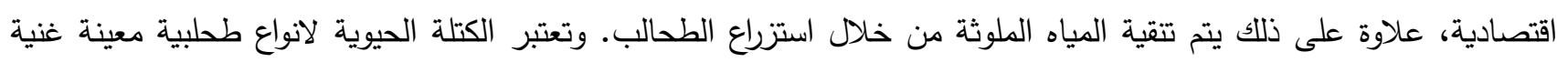


بالسكريات والبروتينات والدهون، وتُعد مصدراً جيداً للمنتجات الحيوية المتتوعة وخزين غذائي للأغذية والأعلاف والوقود الحيوي،

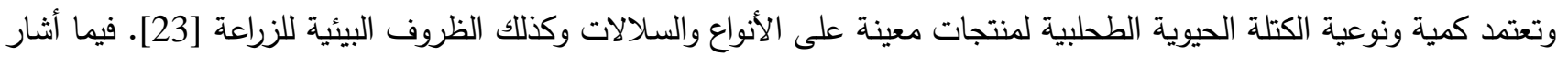
العديد من الباحثين الى اعتبار الطحالب الدقيقة كائنات حية تتتج مجموعة متميزة من المركبات الكيميائية والبيولوجية كالكربوهيدرات والبروتينات والدهون[24] من الباحن الدين

الجدول (3): الكتلة الحيوية الجافة والمحتوى الدهني الكلي للطحالب الدقيقة المدروسة.

\begin{tabular}{|c|c|c|c|}
\hline $\begin{array}{c}\text { الدهون الكلية } \\
\text { \% }\end{array}$ & الجافة غم لتر - الحيوية & اسم الطحلب & ت \\
\hline 23.4 & 1.58 & S. dimorphus & 1 \\
\hline 29.6 & 0.91 & C. vulgaris & 2 \\
\hline 24.2 & 1.39 & Chlo. humicola & 3 \\
\hline
\end{tabular}

تقدير محتوى الدهون الكلية لأنواع الطحالب الدقيقة: فصلت الدهون الكلية عن المكونات الكيميائية الاخرى لخلايا الطحالب بقهع الفصل باستخدام خليط الدذيبات العضوية

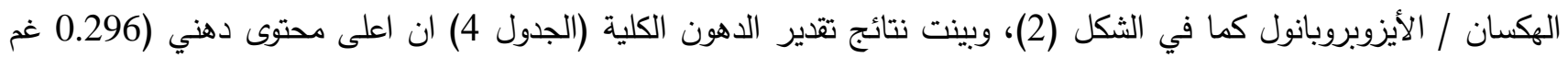

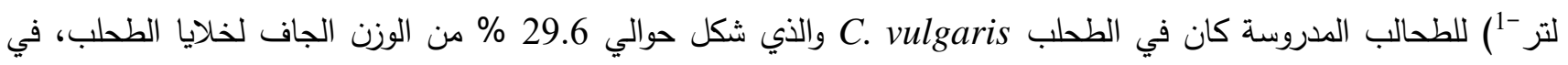
حين بلغ المحتوى الدهني (Chlo. humicola للطحلب. في حين جاء بالمرتبة الثالثة الطحلب S. dimorphus بقيمة المحتوى الدهني (0.234 غم لتر -1) في خلاياه والتي تشكل

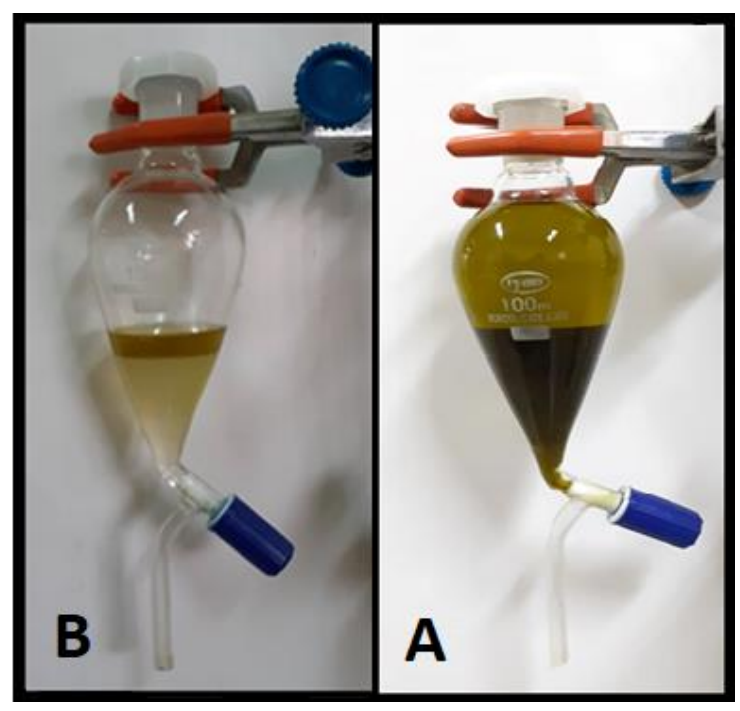

حوالي 23.4 \% من الوزن الجاف.

\begin{abstract}
الثكل (2): فصل الدهون الكلية وفصل
الاحماض الدهنية بثكل حر،

A : فصل الدهون الكلية عن المكونات

الكيميائية الاخوى، النون الهن

B : فصل الدهون المرة بطريقة الصوبنة
\end{abstract}

وقد بينت الدراسات أن من مزايا استخدام الطحالب لإنتاج الوقود الحيوي هي القدرة في السيطرة عليها من خلال تراكم وإفراز

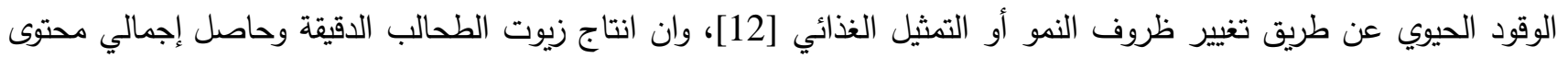

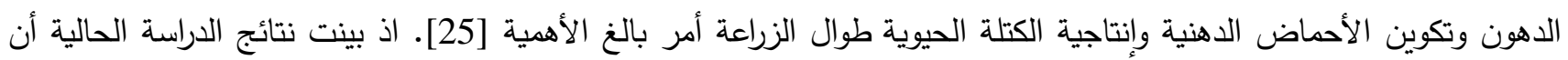

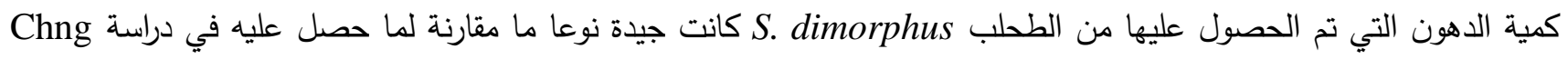
[26] التي وجدت أن نسبة الدهون الكلية في الكتلة الحيوية الجافة للطحلب S. dimorphus كانت نسبة 14 \% ع. كما أظهرت 
النتائج التي حصل عليها Al-Hisenawe [27] من تتمية الطحلب S. obliqus التي لم تتجاوز 13\% فقط من الدهون الكلية. أما فيما يتعلق بالدهون الكلية المنتجة من الطحلب الدقيق C. vulgaris قيد الدراسة فقد أظهرت النتائج أنها كانت جيدة (29.6

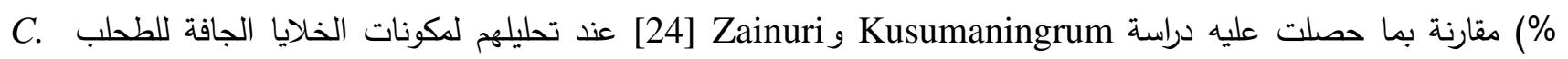
vulgaris

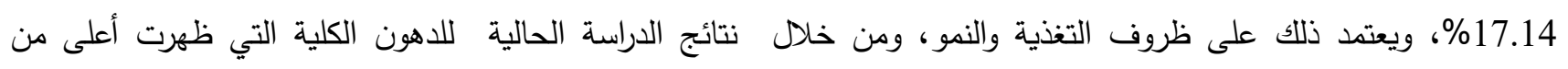
نظيراتها ووصول النمو إلى مرحلة الطور الثابت في الطحلب الدقيق C. vulgaris فان طول فترة الحضانة تلعب ذلك دوراً مهما في بناء الزيوت وبالأخص الدهون الثلاثية المهمة لإنتاج الوقود الحيوي، الا أنه لا يمكن استخدام جميع سلالات الطحالب الدقيقة

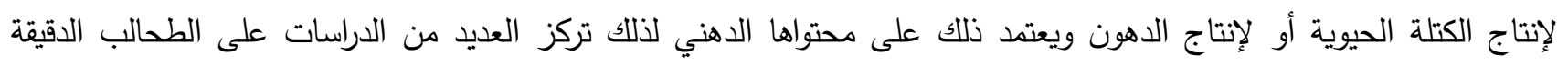
لتحديد إمكاناتها في إنتاج الدهون [29]. وأظهرت النتائج الدهون الخاصة بالطحلب Chlo. humicola بأنها مرتفعة كثيراً عن

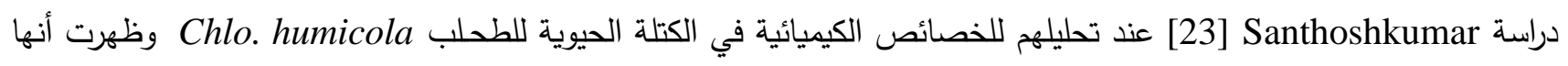

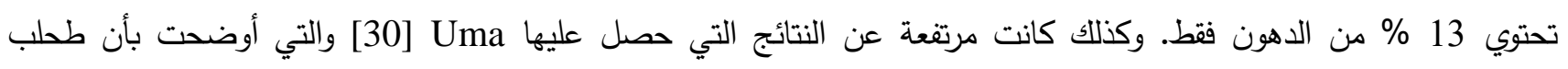
Chlo. humicola

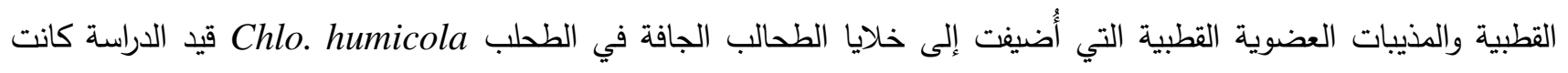
مهمة لضمان استخلاص كامل لجميع الدهون المتعادلة، سواء في تشكيل قطيرات الدهون الحرة في سايتوبلازم الخلية أو تثكيل

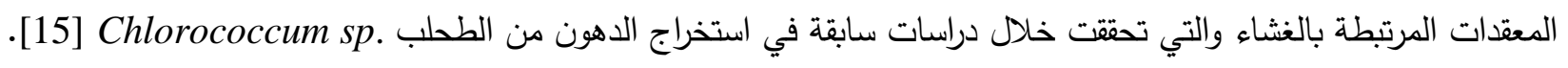
تشخيص الاحماض الدهنية في الطحالب المدروسة بتقنية كروماتوكرافيا الغاز-السائل (GLC):

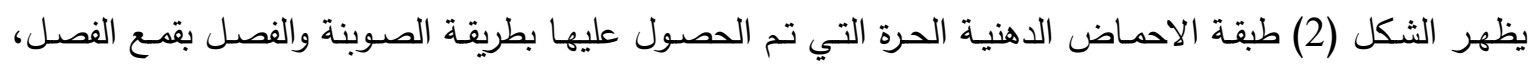
واعتمدت النتائج التي تم الحصول عليها في تشـخيص استرات الاحمـاض الدهنيـة الحرة بتقنية GLC في الطحالب المدروسـة فئسة باستخدام مخططات تحليل استرات للأحماض الدهنية القياسية (الملحق 1) المأخوذة بنفس ظروف الجهاز وبالاعتماد على نسبة الإنة ظهور الاحماض ومساحاتها في العينات المشخصة، ومقارنة زمن احتجاز كل استر دهني مع زمن احتجاز استر الدهون القياسية

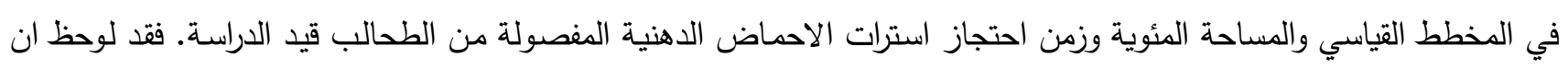
المحتوى الخلوي لطحلب S. dimorphus يحتوي نسب من استرات الاحماض الدهنية والمبينة في الجدول (4) والملحق (2)،

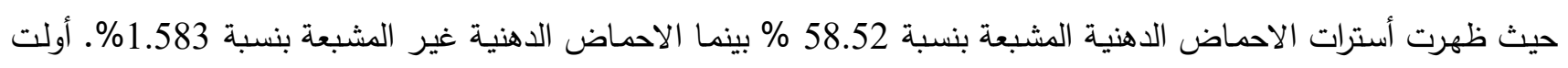
الكثير من الدراسات بالأحماض الدهنية الحرة والتي تُعتبر العنصر الاساسي والمؤثر لاحتراق الوقود الحيوي، حيث يختلف تركيبها

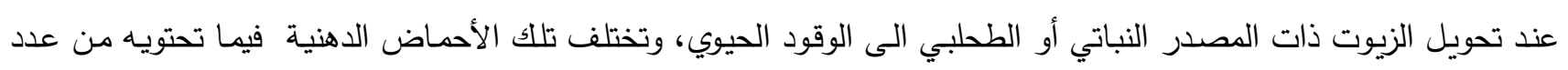

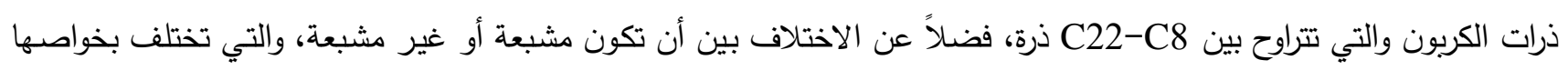

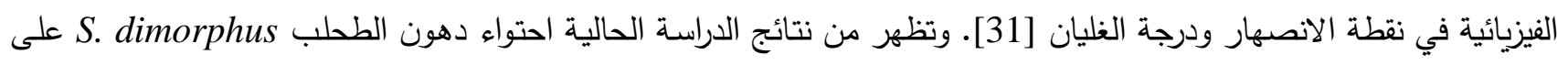

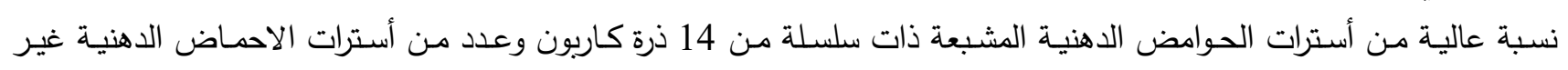
المشبعة وكمية ضئيلة جداً من أسترات الأحماض الدهنية الأحادية عدم التشبع والتي تشير الى أهمية طول سلسلة ذرات الكاربون

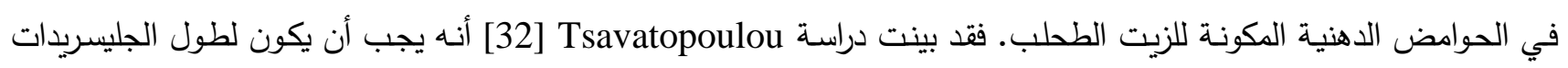
الأكثر ملاءمة لإنتاج الوقود الحيوي طول سلسلة ما بين C14 و C22 ودرجة منخفضة من أسترات الحوامض الدهنية عديمة التشبع

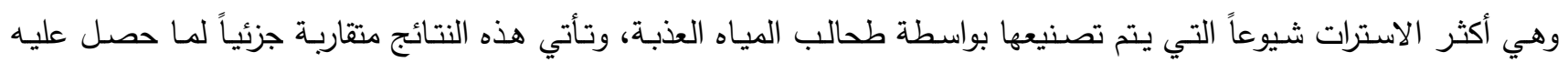

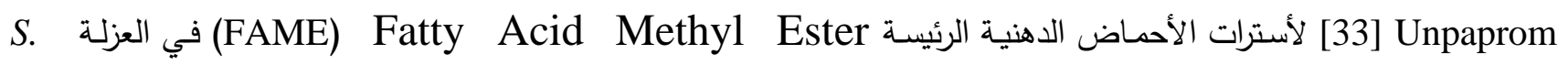
acuminatus

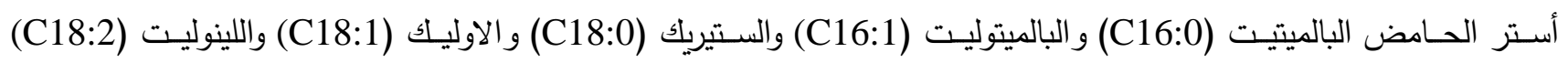


واللينولينت (C18:3) بنسبة عالية من إجمالي محتوى FAME، بخلاف احتواء الطحلب قيد الدراسة على أعلى نسبة من أستر

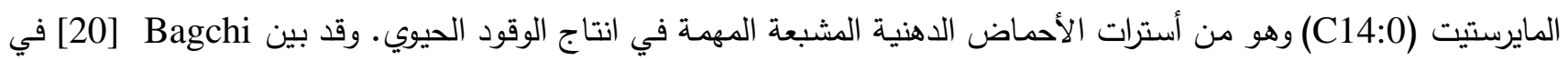
دراستهم الى أن تحديد محتويات الدهون في الوقود الحيوي المنتج من الطحلب S. obliquus GA 45 خلال التغيرات الموسمية قد

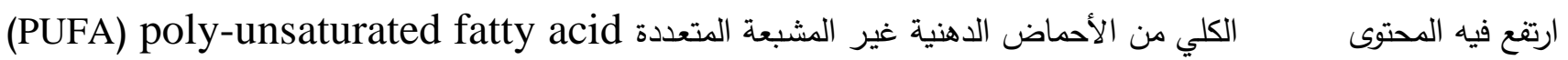

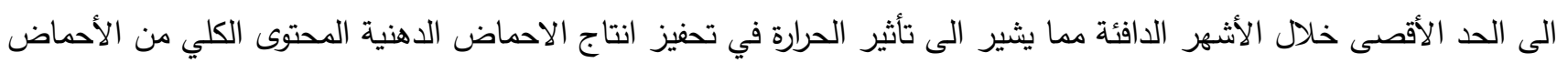

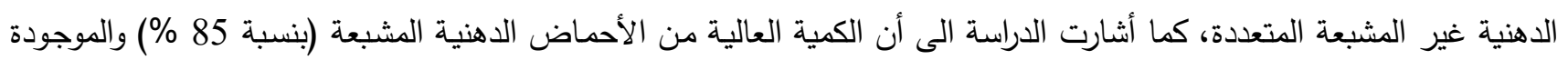

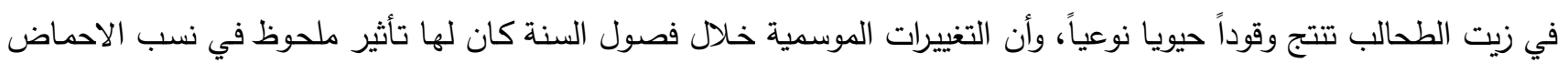
الدهنية المشبعة وغير المشبعة تبعا لتغير الظروف الجوية.

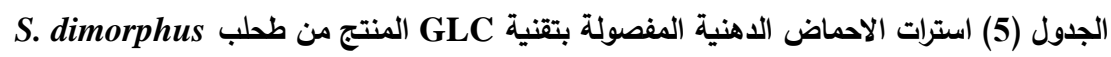

\begin{tabular}{|c|c|c|c|c|c|c|}
\hline زالحن احتجاز & زمن احتجاز & الدامضي & 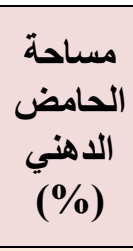 & ذرات & الحامض الدهني & $\ddot{~}$ \\
\hline 6.015 & 7.094 & 2.036 & 0.2941 & C6:0 & Hexanoic (caproic)acid & 1 \\
\hline 15.578 & 15.255 & 47.105 & 6.8052 & C14:0 & Myristic acid & 2 \\
\hline 16.098 & 16.341 & 1.359 & 0.1964 & $\mathrm{C} 14: 1$ & Myristoleic acid & 3 \\
\hline 17.785 & 17.574 & 0.547 & 0.0792 & C16:0 & Palmatic acid & 4 \\
\hline 20.731 & 19.818 & 1.639 & 0.2368 & C18:0 & Stearic acid & 5 \\
\hline 21.959 & 22.815 & 0.224 & 0.0323 & C18:2 & Linoleic acid & 6 \\
\hline 29.456 & 29.322 & 7.193 & 1.0392 & $\mathrm{C} 24: 0$ & Lignoceric acid & 7 \\
\hline \multicolumn{2}{|c|}{ ألاحماض الدهنية الكلية (\%) } & 60.103 & 8.6832 & \multicolumn{3}{|c|}{ مجموع مساحات الاحماض الدهنية في العينة } \\
\hline \multicolumn{2}{|c|}{ المتبقيات (\%) } & 39.896 & 5.7638 & \multicolumn{3}{|c|}{ مجموع مساحات المتبقيات في العينَة } \\
\hline \multicolumn{2}{|c|}{ الدهن الكلي للعينة (\%) } & 99.999 & 14.447 & \multicolumn{3}{|c|}{ المجموع الكلي لمساحات العينة } \\
\hline
\end{tabular}

أما المحتوى الدهني الخلوي للطحلب C. vulgaris فقد فتم تشخيص عدد من استرات الاحماض الدهنية والمبينة في الجدول (5) والملحق (3) اذ ظهرت استرات الاحماض الدهنية المشبعة بنسبة 26.94\% بينما كانت استرات الاحماض الدهنية الغير مشبعة بنسبة 13.178\%، ومن هذه النتائج تبين أن النسبة الأكبر التي تم الحصول عليها من أسترات الاحماض الدهنية

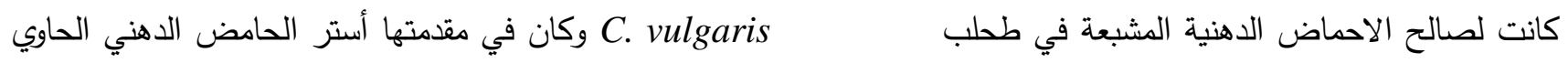
على 16 ذرة كاربون ونسبة قليلة من أسترات الاحماض الدهنية المشبعة الاخرى، بينما ظهرت نسبة أسترات الاحماض الدهنية الاحادية عديمة التشبع قليلة جداً. ويعزى ظهور أستر البالمتيت الحاوي على 16 ذرة كاربون بنسبة عالية مقارنة ببقية الأسترات المشبعة وغير المشبعة الى التوافق في النتائج التي توصل اليها العديد من الدراسات التي تشير الى أن أستر البالميتيت (C16:0) من الأسترات المهمة في الطحالب الدقيقة. فقد أكد Stansell [34 أن محتوى الدهون المستخدمة لإنتاج الوقود الحيوي يتكون أساساً من أسترات الأحماض الدهنية (C16:0) و (C18:1). كما ذكر Nautiyal [35] أن المكون الرئيس الموجود من بين أسترات الأحماض الدهنية للطحالب الدقيقة هو البالمتيت (C16:0 (C16) وبنسبة حوالي 35 \% \% C. و Zusumaningrum vulgaris اضافة لوجود أسترات الحوامض C22-C20 المشبعة وغير المشبعة والعديدة عدم التشبع، كما ظهرت النتائج متوافقة لما أشار اليه Hempel [36] بأن هناك تركيزاً منخفضاً للغاية من أسترات الحامض اللينوليت (C18:2) واللينولينت (C18:3) وبلغت 1.41\% و 0.19 \% في دهون الطحلب .Chlorella sp على التوالي. 
الجدول (5): استرات الاحماض الدهنية المفصولة بتقنية GLC المنتج من طحلب Chlorella vulgaris

\begin{tabular}{|c|c|c|c|c|c|c|}
\hline $\begin{array}{c}\text { زالدن احتجاز } \\
\text { الدامضي القياسي } \\
\text { (دقيقة) }\end{array}$ & زمن احتجاز & 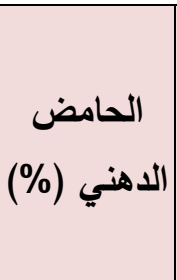 & الحامض & ذالكربون & مض الدهني & \\
\hline 6.015 & 7.090 & 0.4170 & 0.0953 & C 6:0 & Cap & \\
\hline 15.578 & 15.485 & 0.1950 & 0.0444 & C 14:0 & c acid & - \\
\hline 17.785 & 17.154 & 25.906 & 5.9227 & C 16:0 & Palmatic acid & 3 \\
\hline 20.216 & 20.026 & 0.1550 & 0.0355 & C 18:0 & Stearic acid & 4 \\
\hline 21.959 & 21.690 & 0.0930 & 0.0213 & C 18:2 & Linoleic acid & 5 \\
\hline 23.755 & 23.644 & 0.1140 & 0.0260 & C 20:0 & Arachidic acid & 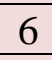 \\
\hline 42.407 & 24.269 & 13.085 & 2.9916 & C 20:4 & Arachidonic acid & 7 \\
\hline 29.456 & 29.343 & 0.1510 & 0.0346 & C 21:0 & Lignoceric acid & 0 \\
\hline \multicolumn{2}{|c|}{ الاحماض الدهنية الكلية (\%) } & 40.116 & 9.1714 & \multicolumn{3}{|c|}{ مجموع مساحات الاحماض الدهنية في العينة } \\
\hline \multicolumn{2}{|c|}{ المتبقيات (\%) } & 59.883 & 13.6904 & \multicolumn{3}{|c|}{ مجموع مساحات المتبقيات في العينة } \\
\hline \multicolumn{2}{|c|}{ الدهن الكلي للعينة (\%) } & 99.999 & 22.8618 & \multicolumn{3}{|c|}{ المجموع الكلي لمساحات العينة } \\
\hline
\end{tabular}

فيما بينت أسترات الاحماض الدهنية المشخصة للطحلب Chlo. humicola والمبينة في الجدول (6) والملحق (4) ظهور استرات الاحماض الدهنية المشبعة بنسبة 47.71\% بينما كانت نسبة الاحماض الدهنية الغير مشبعة الظاهرة 28.44\%. وتشير هذه النتائج ان نسبة أسترات الاحماض الدهنية المشبعة في طحلب Chlo. humicola تثكل النسبة الأكبر من مجموع الأحماض

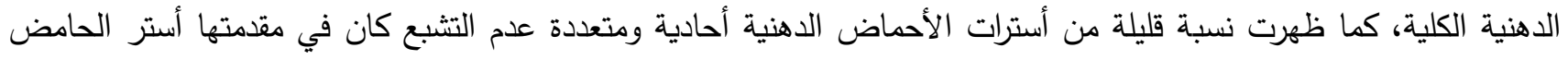
الدهني غير المشبع الحاوي على 18 ذرة كاربون مع نسبة ضئيلة جداً من أسترات الأحماض الدهنية غير المشبعة الأخرى. كما

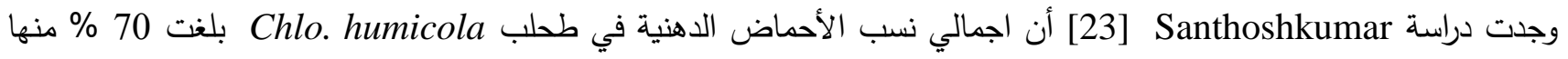
أحماض دهنية أحادية متشبعة، و17.4 \% من أحماض دهنية أحادية عديمة الثبع و2.12 \% من من أحماض دهنية غير مشبعة متعددة. واظهرت نتائج الدراسة الحالية أن هناك نسبة عالية من أستر الحامض الدهني المشبع الاركيديت (C20:0) والذي يقع دنيه

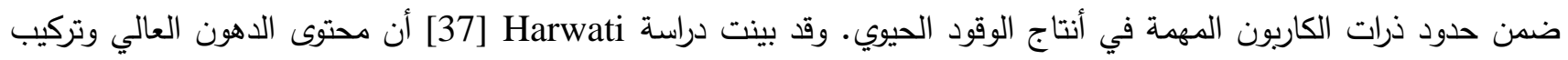

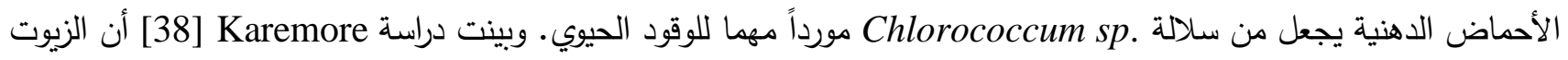
التي تحتوي على نسبة عالية من الأحماض الدهنية المشبعة لها استقرار تأكسدي جيد مما يساعد في عملية لتخزين أطول، ولا يمكن

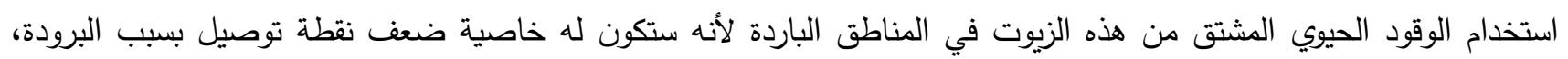

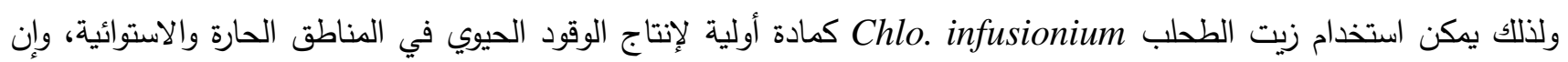

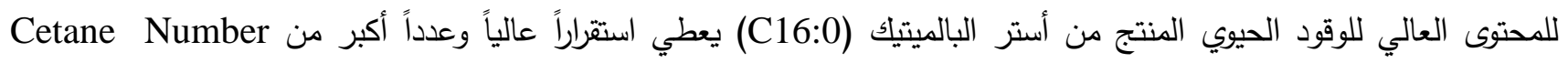
وذللك لأن الأحماض الدهنية المشبعة ذات عدد ذرات الكربون C22-C12 والتي تستخرج منها أسترات الحامض الدهني تعمل على زيادة في عدد Cetane وهو مؤشر لضمان سرعة حدوث احتراق الوقود الحيوي والضغط اللازم للاشتعال وهو عامل مهم في تحديد نوعية الوقود من خلال تكوين الدخان الأبيض الأقل ضرراً وتقليل المستوى المرتفع لانبعاثات أكاسيد النيتروجين وتحسين لعندين استقرار الوقود الحيوي [32]. 
Chlo. humicola الجدول (6) GLC المنتج من طحلب البرات الاحماض الدهنية المفصولة بتقنية

\begin{tabular}{|c|c|c|c|c|c|c|}
\hline زالحن احتجاز & زمن احتجاز & الحامض & الدامضاحة & ذرات & الحامض الدهني & $ت$ \\
\hline 6.015 & 6.110 & 0.8990 & 0.1003 & C 6:0 & Caproic acid & 1 \\
\hline 15.578 & 15.074 & 0.0340 & 0.0037 & C 14:0 & Myristic acid & 2 \\
\hline 16.098 & 16.594 & 0.0740 & 0.0083 & C 14:0 & Myristoleic acid & 3 \\
\hline 17.785 & 17.439 & 7.4280 & 0.8282 & C 16:0 & Palmatic acid & 4 \\
\hline 20.216 & 20.112 & 0.0310 & 0.0035 & C 18:0 & Stearic acid & 5 \\
\hline 21.959 & 22.142 & 28.371 & 3.1636 & C $18: 2$ & Linoleic acid & 6 \\
\hline 25.353 & 24.970 & 0.0530 & 0.0059 & C $18: 3$ & Linolenic acid & 7 \\
\hline 23.755 & 23.284 & 36.205 & 4.0372 & C 20:0 & Arachidic acid & 8 \\
\hline 24.407 & 24.175 & 0.1530 & 0.0170 & C 20:4 & Arachidonic acid & 9 \\
\hline 26.045 & 26.831 & 0.0143 & 0.0016 & C 22:1 & Erucic acid & 10 \\
\hline 29.456 & 29.341 & 0.0404 & 0.0045 & C 24:0 & Lignoceric acid & 11 \\
\hline \multicolumn{2}{|c|}{ الاحماض الدهنية الكلية (\%) } & 73.302 & 8.1740 & \multicolumn{3}{|c|}{ مجموع مساحات الاحماض الدهنية في العينة } \\
\hline \multicolumn{2}{|c|}{ المتبقيات (\%) } & 26.697 & 2.9770 & \multicolumn{3}{|c|}{ مجموع مساحات المتبقيات في العينة } \\
\hline \multicolumn{2}{|c|}{ الدهن الكلي للعينة (\%) } & 99.999 & 11.151 & \multicolumn{3}{|c|}{ المجموع الكلي لمساحات العينة } \\
\hline
\end{tabular}

الملحق Supplement:

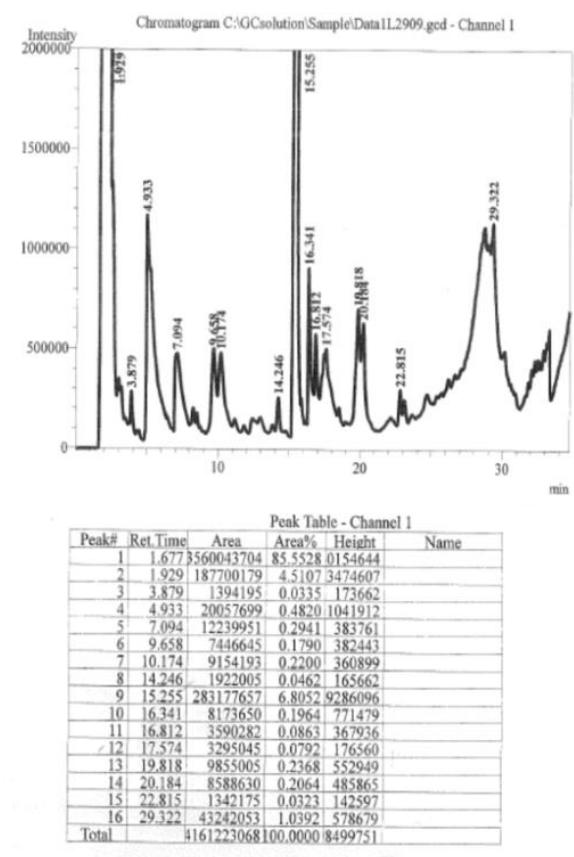

الملحق (2): أسترات الاحماض الدهنية المفصولة من

والمشخصة S. dimorphes الخلايا الجافة لطحلب

GLC
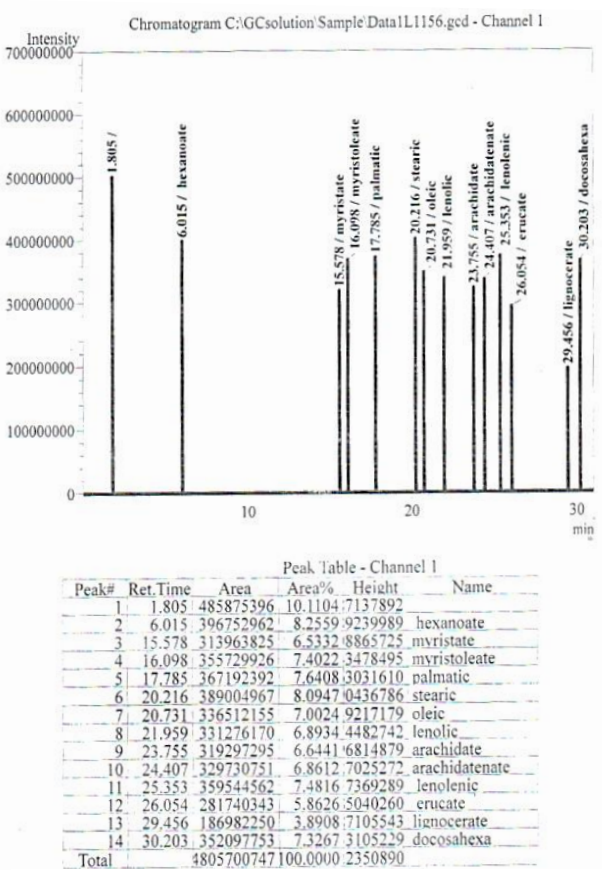

الملحق (1) أسترات الاحماض الاهنية

GLC 


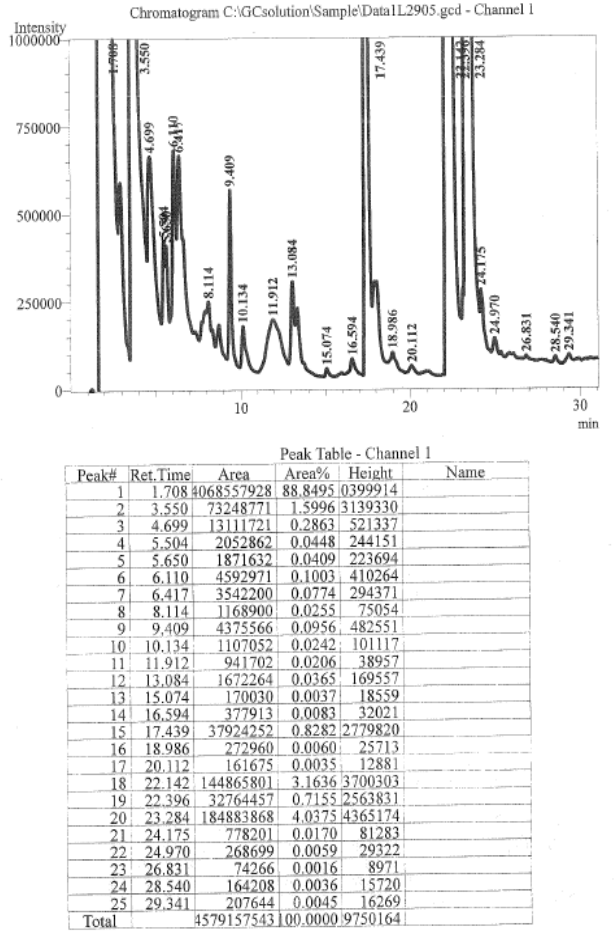

أسترات الاحماض الدهنية المفصولة من :الملحق (4) والمشخصة Chlo. humicola (الخلايا الجافة لطحلب GLC
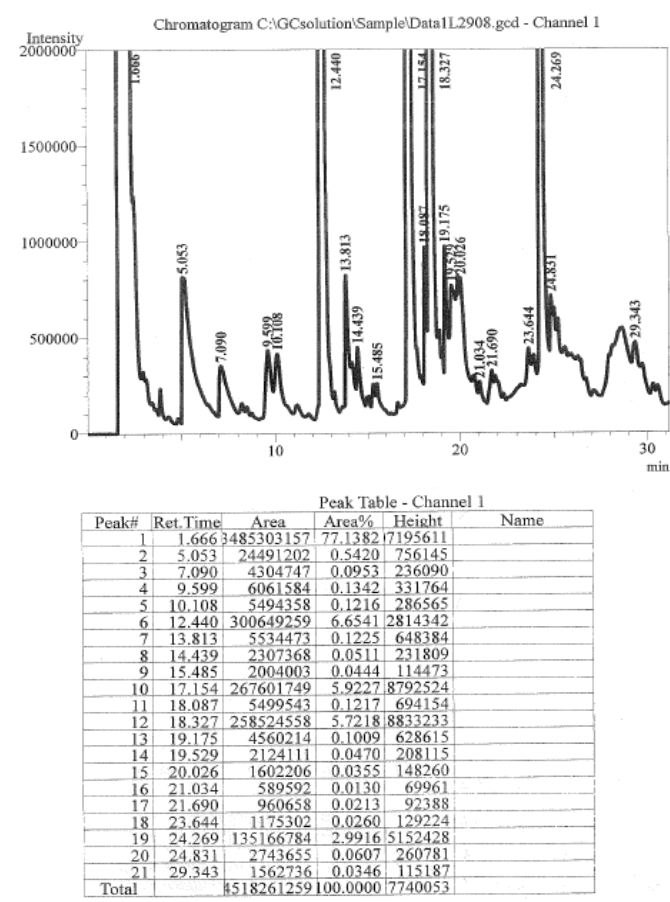

الملحق (3): أسترات الاحماض الدهنية المفصولة والمشخصة Vulgaris من الخلايا الجافة لطحلب GLC

Conclusions الاستنتاجات

اظهـرت الدر اسـة الحاليـة نجاحاً في تتمية الطحالب الدقيقة Chlo. humicola و C. vulgaris ، S. dimorphus باستخدام مفاعل ضوئي محلي الصنع سعة 5 لتر وبواقع 3 لتر من الوسط الزرعي بعد 25 يوماً من التحضين، وانتاج كتلة حيوية جيدة في

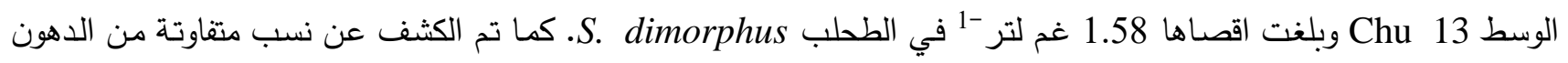

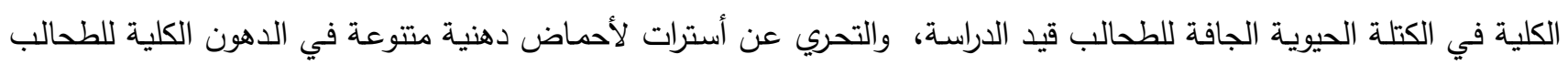
والتي كانت افضلها في الطحلب الدقيق S. dimorphus اذ كانت نسبة استرات الاحماض الدهنية 58.52 \% بينما الاحماض فئس الدهنية غير المشبعة بنسبة 1.583\% والتي تمثل قيمة جيدة من الاحماض الدهنية في تركيب الوقود الحيوي مقارنة بنسب استرات الاحماض الدهنية في الطحالب الاخرى قيد الدراسة وتشير هذه النسبة لأهمية زيادة نسب استرات الاحماض الدهنية المشبعة التي فئي ترفع من عدد Cetane وهو مؤشر مهم لضمان سرعة حدوث احتراق الوقود الحيوي وهو عامل مهم في تحديد نوعية الوقود من خلال تكوين الدخان الأبيض الأقل ضرراً وتقليل المستوى المرتفع لانبعاثات أكاسيد النيتروجين وتحسين استقرار الوقود الحيوي. الشكر والتقدير:نتقدم بخالص الثكر والعرفان لرئاسة جامعة الموصل لدعمها المتواصل للباحثين والسماح لنا بالعمل في مختبرات الجامعة. References المصادر

[1] Kotasthane, T.. Mar. Sci. Res. \& Dev. 7(2) (2017).

[2] Williams, P.J.L., Laurens, L.M.L. . Ene. Env. Sci. 3, 554-590 (2010).

[3] Chisti,Y.. Biotechnol Advances. 25(3), 294-306 (2007).

[4] Bansal, B. K. and Sharma, M. P. Renew. and Sust. Ene. Rev., 9 (4) 363- 378(2005).

[5] Mata, T.M, Antonio A., Martina, N. and S. Caetano. Renew. and Susta. Ene. Rev. 14: 217232(2010). 
[6] Alam, F., Mobin, S. and H. Chowdhury. Proc. Eng. 105: 763 - 768. (2015).

[7] Yamaguchi,K.;Nakano,H.;Murakami,M.;Kansu,S.;Nakayama,O.;Kanda,M.;Na kmura, A. and Iwamoto, H . Agric. Biol. Chem. 51 :493-498.(1987).

[8] Kais, Md. I., Chowdhury, F. I.and Shahriar, K. F. .World Renew. Ene. Cong. 8-13 May ,Sweden. Bioen. Tech. (2011).

[9] Li, X., Pribyl, P., Bisova, K., Kawano, S. and Cepak, V. . Biotech. and Bioeng., 110(1) (2018).

[10] Ahmed, F., Khan, A. U. and Yasar, Abdulllah. Afri. J. Env. Sci. Tech.7 (6):358-364 (2013).

[11] Mohamed, S. and Nedtham, A. A. .J of Albaath Univ. 37(6) 103-125 (2015). (In Arabic)

[12] Almutairi, A. PhD. Thesis Dept. of Biology and Biotechnology.. Univ. of Sheffield, UK. (2015).

[13] Alekeli. T.M. A. PhD. Thesis. College of Education. for Pure Sciences. Ibn Al Haitham . Baghdad, Iraq (2016) (In Arabic).

[14] Rios, S.. Thesis. Department d'Enginyeria Química. Univ. Roviral Virigli. Tarragona: T 5402014 (2012).

[15] Halim, R.; Danquah, M. K. and Webley, P. A. J. Biotech. Advan., 30 (3), 709-732 (2012).

[16] Yadavalli,R.;Rao,R.S. and Rao,C.S. Inter. J. Eng. Res. Appl.. 2 (3) 2446-2453 (2012).

[17] Arthur, I. Vogel. Practical Organic Chemistry Including Qualitative Organic Analysis, $3^{\text {rd }}$ ed., p.445 (1972).

[18] Loury, MA . Rev. France. Corps. Gras., 14: 383-9 (1967).

[19] Pugliese, A.; Biondi, L. and Bartocci, P. Ferm.6(46)(2020).

[20] Bagchi, S. K.; Patnaik, R.; Sonkar, S.; Koley, S.; Rao, P. S. and Mallick, N. . Renew. Ene., 139, 976-987 (2019).

[21] Ferro, L.; Gentili, F. G. and Funk, C. . Algal Res. 32, 44-53(2018).

[22] Debowski, M. ; 'nski, M. Z. ; Kisielewska, M. ; Kazimierowicz , J.; Dudek, M. ; Swica, I. and Rudnicka, A.. Proc., 8(5) 1-14 (2020).

[23] Santhoshkumar, K.; Prasanthkumar S. and J. G. . J. Plant Stud., 5(1) p.48 (2016).

[24] Kusumaningrum, H. P. and Zainuri, M. . J. of Foo. Proc. Tech. 9(1)1-5 (2018).

[25] Rosenberg, J. N.; Kobayashi, N.; Barnes, A.; Noel, E. A.; Betenbaugh, M. J. and Oyler, G. A. Plos. One., 9(4) (2014).

[26] Chng, L. M.; Lee, K. T. and Chan, D. J. C. Ene. Con. Manag. 141, 410-419. (2016).

[27] Al-Hisenawy, S. H. A. MSc. Thesis. Biology Dept. College. of Sciences. Univ. of Thi-Qar. Iraq (2016). (In Arabic)

[28] Bi, Z, and He B. B. .America Soc. Agri. Biol. Eng., 56, 1529-1539 (2013).

[29] Ngoc, L. D. B.; Adenan, M. F.; Bato, B. A.; Mansor, N. and Mahadzir, S. . Inter. J. of Chem.l Eng. and Appl. 4(4) 262-265(2013).

[30] Uma, R. ;Sivasubramanian V. and Devaraj, S. N. Indian. J. Pharm. Sci. Res., 5(1) 19-22(2015).

[31] Abo-Alnaja, H.. Acad. Publishing Library. Egyp. joint stock company. The Arab Republic of Egypt. (2011) (In Arabic)

[32] Tsavatopoulou, V. D.; Aravantinou, A. F. and Manariotis, I. D. J. Chem. Tech. Biotech. 95, 2421-2429 (2020).

[33] Unpaprom, Y.; Tipnee, S. and Rameshprabu, R. . Int. J. Sustain. Green. Energy., 4(1) 16(2015).

[34] Stansell, G. R.; Gray, V. M. and Sym, S. D. J. Appl. Phyco., 24, 791-801(2012).

[35] Nautiyal, P.; Subramanian K. A. and Dastidar M.G. Fuel Proc. Tech., 120, 79-88(2014).

[36] Hempel, N.; Petrick, I. and Behrendt, F. J. Appl. Phyco., 24, 1407-1418. (2012).

[37] Harwati, T. U.; Willke, T. and Vorlop, K. D.. Biores. Tech. 121, 54-60 (2012).

[38] Karemore, A.; Pal, R. and Sen, R.. Algal Res. 2(2) 113-121 (2013). 